\title{
Conhecimento e conservação dos peixes marinhos e estuarinos (Chondrichthyes e Teleostei) da costa norte do Brasil
}

\author{
Alexandre Pires Marceniuk ${ }^{1,2,5}$, Rodrigo Antunes Caires $^{3}$, Wolmar Benjamin Wosiacki ${ }^{2}$ \& Fabio Di Dario ${ }^{4}$ \\ ${ }^{1}$ Instituto de Estudos Costeiros, Universidade Federal do Pará - UFPA, Campus de Bragança, Alameda \\ Leandro Ribeiro, s/n, CEP 68600-000, Bragança, PA, Brasil \\ ${ }^{2}$ Setor de Ictiologia, Museu Paraense Emílio Goeldi, CP 399, CEP 66040-170, Belém, PA, Brasil \\ ${ }^{3}$ Museu de Zoologia, Universidade de São Paulo-USP, CP 42494, CEP 04218-970, São Paulo, SP, Brasil \\ ${ }^{4}$ Núcleo em Ecologia e Desenvolvimento Socioambiental - NUPEM, Universidade Federal do Rio de \\ Janeiro - UFRJ, Campus Macaé, CP 119331, CEP 27910-970, Macaé, RJ, Brasil \\ ${ }_{5}^{5}$ Autor para correspondência: Alexandre Pires Marceniuk, e-mail: a_marceniuk@hotmail.com
}

MARCENIUK, A.P., CAIRES, R.A., WOSIACKI, W.B. \& DI DARIO, F. Knowledge and conservation of the marine and estuarine fishes (Chondrichthyes and Teleostei) of the north coast of Brazil. Biota Neotrop. 13(4): http://www.biotaneotropica.org.br/v13n4/en/abstract?inventory+bn02613042013

\begin{abstract}
The tropical western South Atlantic, which includes a substantial portion of the Brazilian Exclusive Economic Zone, is a region of endemism broadly recognized as being of prime importance for the conservation of the marine biodiversity. The north coast of Brazil, which comprises the states of Amapá, Pará and Maranhão from the mouth of the rio Oiapoque to the mouth of the rio Parnaíba, harbors the largest continuous mangrove in the world, with approximately $8,900 \mathrm{~km}^{2}$. The high discharge of freshwater and continental sediments in the delta of the Amazonas affects the regime of tides, ocean currents, and several oceanographic processes of the north coast, with direct impact on the composition of the biota found in the region. Despite its economic value and intrinsic biological relevance, several aspects of the diversity of the marine and estuarine fishes of the region are poorly known. This situation results mainly from a historical imbalance in terms of the number of studies devoted to increasing the knowledge of the marine biota along the Brazilian coast, such as those dealing with species inventory and taxonomic revisions, which are typically concentrated in the south and southwestern portions of the country. The scientific production focused on marine organisms of the north coast is also imbalanced, and reflects the relatively small number of taxonomists and research groups working on that subject. The insufficient knowledge of the biodiversity of the marine and estuarine fishes of the north coast is an impediment to the implementation of adequate public policies aimed at the management of natural resources in the region. In the long term, that situation is potentially harmful in terms of conservation of a still poorly known biota. A better understanding of the marine fish fauna of the north coast of Brazil will be achieved only through the investment in scientific research and personnel training in systematics and biogeography, coupled with the modernization of the current infrastructure and expansion of scientific collections of the region.
\end{abstract}

Keywords: biodiversity, inventory, natural resources, zoological collections, Exclusive Economic Zone.

MARCENIUK, A.P., CAIRES, R.A., WOSIACKI, W.B. \& DI DARIO, F. Conhecimento e conservação dos peixes marinhos e estuarinos (Chondrichthyes e Teleostei) da costa norte do Brasil. Biota Neotrop. 13(4): http://www.biotaneotropica.org.br/v13n4/pt/abstract?inventory+bn02613042013

Resumo: A região tropical do Atlântico Sul ocidental, que inclui uma porção substancial da Zona Econômica Exclusiva brasileira, é uma região de endemismo amplamente reconhecida como sendo de importância primária para a conservação da biodiversidade marinha. A costa norte do Brasil, que inclui os Estados do Amapá, Pará, e Maranhão, desde a foz do rio Oiapoque até a foz do rio Parnaíba, abriga o maior manguezal contínuo do mundo, com aproximadamente $8.900 \mathrm{~km}^{2}$. A alta descarga de água doce e sedimentos continentais no delta do Amazonas afeta o regime das marés, correntes oceânicas, e uma série de processos oceanográficos da costa norte, com impactos diretos na composição da biota encontrada na região. Apesar de seu valor econômico e relevância biológica intrínseca, diversos aspectos da diversidade de peixes marinhos e estuarinos da região são pouco conhecidos. Esta situação resulta principalmente de um desequilíbrio histórico em termos do número de estudos voltados ao conhecimento da biota marinha ao longo da costa brasileira, como aqueles que tratam do inventariamento de espécies e revisões taxonômicas, que são tipicamente concentrados nas porções sul e sudeste do país. A produção científica focalizada nos organismos marinhos da costa norte também é desequilibrada e reflete o número relativamente baixo de taxonomistas e grupos de pesquisa trabalhando com o tema. O conhecimento insuficiente da biodiversidade dos peixes marinhos e estuarinos da costa norte é um impedimento à implantação de políticas públicas adequadas voltadas para o manejo dos recursos naturais na região. À longo prazo, essa situação é potencialmente prejudicial em termos da conservação de uma biota ainda pouco conhecida. Um melhor conhecimento da fauna de peixes marinhos da costa norte do Brasil será atingido apenas através do investimento em pesquisas científicas e formação de pessoal em sistemática e biogeografia, acoplado à modernização da infraestrutura e expansão das coleções científicas da região.

Palavras-chave: biodiversidade, inventariamento, recursos naturais, coleções zoológicas, Zona Econômica Exclusiva. 


\section{Introdução}

O termo biodiversidade tem sido amplamente utilizado por biólogos, ambientalistas, líderes políticos e cidadãos comuns desde meados da década de 1980. A popularização do termo coincide com o aumento da degradação do meio ambiente e a perda de espécies nas últimas décadas do século XX, quando foram reconhecidas as regiões mais ricas biologicamente, ou hotspots de biodiversidade (Myers et al. 2000). A porção tropical do Atlântico Sul ocidental, incluída na Zona Econômica Exclusiva brasileira (ZEE), é uma importante área de endemismo marinho e uma área prioritária para conservação da biodiversidade (McKenna \& Allen 2002). Entretanto, a região permanece insatisfatoriamente conhecida em relação a sua diversidade biológica (Couto et al. 2003, Amaral \& Jablonski 2005).

A costa brasileira possui aproximadamente $8.500 \mathrm{~km}$ de extensão, do rio Oiapoque (AP) ao Arroio Chuí (RS), com uma grande diversidade de características geológicas, geográficas, climáticas, hidrográficas e sedimentológicas, que constituem uma grande diversidade de ambientes, como estuários, lagoas, lagunas, praias arenosas, costões rochosos e recifes de coral (Brasil 2007). Ekau \& Knoppers (1999), com base nestas variáveis, reconheceram cinco regiões no litoral brasileiro: região Norte, da foz do rio Oiapoque $\left(4^{\circ} 16^{\prime} \mathrm{N}\right)$, no Amapá, à foz do rio Parnaíba ( $\left.3^{\circ} \mathrm{S}\right)$, no Maranhão; região Nordeste, da foz do rio Parnaíba à baía de Todos os Santos $\left(13^{\circ} \mathrm{S}\right)$, na Bahia; região Leste, da baía de Todos os Santos ao cabo de São Tomé ( $\left.22^{\circ} \mathrm{S}\right)$, no Rio de Janeiro; região Sudeste, do cabo de São Tomé, ao cabo de Santa Marta (28 40' S), em Santa Catarina; e região Sul, do cabo de Santa Marta ao arroio Chuí (34 ${ }^{\circ}$ $45^{\prime}$ S), no Rio Grande do Sul. Nestas regiões, os traços distintivos da biodiversidade são determinados por fenômenos climatológicos e oceanográficos (Amaral \& Jablonski 2005), que combinados ao componente filogenético (histórico), influenciam o conjunto de espécies encontradas nestas regiões (e.g. Nelson \& Platnick 1981).

A costa norte do Brasil compreende os estados do Amapá, Pará e Maranhão, estendendo-se por mais de $2.500 \mathrm{~km}$, com uma linha da costa bastante diversa (Isaac \& Barthem 1995) (Figura 1). No Amapá, a linha da costa é retilínea, enquanto no nordeste do Pará e noroeste do Maranhão, é profundamente recortada, tornando-se novamente retilínea a partir da baía do Tubarão, no Maranhão (El-Robrini et al. 1992). A região é altamente instável em termos geomorfológicos em decorrência da ação de erosão, com grande deposição de sedimentos que contribuem para o desenvolvimento de planícies alagadas e grande número de ilhas. As reentrâncias e recortes da linha costeira favorecem a existência de ecossistemas estuarinos, dominados pela vegetação de manguezal. Os estados do Amapá, Pará e Maranhão abrigam a maior área de manguezal contínuo do planeta, com cerca de $8.900 \mathrm{~km}^{2}$, que fornece uma grande quantidade de nutrientes para as águas marinhas adjacentes (Dittmar 1999). Os Golfões Marajoara e Maranhense representam complexos estuarinos bastante dinâmicos, com elevada descarga de sedimento de origem fluvial. Somente o rio Amazonas descarrega no oceano entre 80.000 e $250.000 \mathrm{~m}^{3}$ de água doce por segundo (Isaac \& Braga 1999) e até 1,4 milhão de toneladas anuais de sedimentos (Milliman \& Meade 1983), contribuindo para a formação de um substrato predominantemente lamoso na faixa costeira e na plataforma continental interna. A descarga de um volume tão elevado de água doce no ambiente marinho resulta em um sistema dinâmico e altamente energético, interferindo nas condições das marés e ondas, alterando a influência das correntes marinhas,

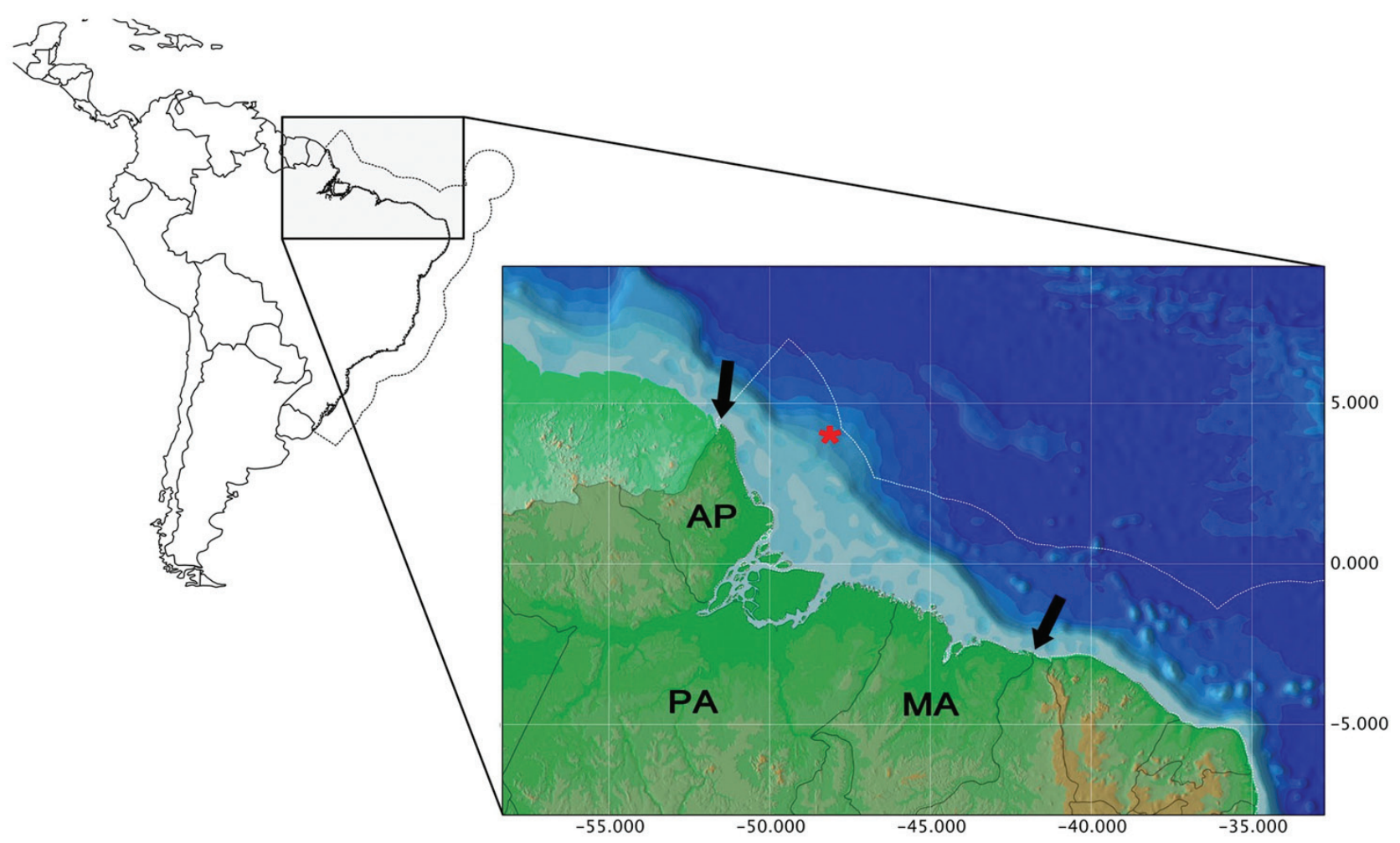

Figura 1. Costa norte do Brasil, ressaltando as fronteiras entre os Estados do Amapá (AP), Pará (PA) e Maranhão (MA). Setas indicam a foz dos rios Oiapoque (norte) e Parnaíba (sul). Estrela vermelha indica o Cone do Amazonas. Linha pontilhada indica o limite da Zona Econômica Exclusiva brasileira. Coordenadas geográficas em Graus Decimais (DD).

Figure 1. North coast of Brazil, highlighting boundaries between the States of Amapá (AP), Pará (PA) and Maranhão (MA). Arrows indicate mouth of rivers Oiapoque (north) and Parnaíba (south). Red star indicates the Amazon Cone. Dotted lines indicate the limit of the Brazilian Exclusive Economic Zone. Geographic coordinates in Decimal Degrees (DD). 
e atuando em uma série de processos oceanográficos, com reflexo direto na composição e distribuição dos recursos naturais (Costa \& Figueiredo Junior 1998).

O Cone do Amazonas, localizado na plataforma continental adjacente à foz do rio Amazonas, é formado pelo acúmulo de sedimentos oriundos do rio Amazonas, que ultrapassa os limites da Zona Econômica Exclusiva. Na região do Cone do Amazonas não existe um talude ou elevação continental típicos, definidos por gradientes de profundidades. Ao invés disso, existe um talude contínuo que se estende até a planície abissal, interrompido por paleocanais e outras feições geológicas construtivas ou erosivas de origem não tectônica (Alberoni et al. 2007). Em relação às correntes marinhas, a costa norte é influenciada pela Corrente Norte do Brasil, ou Corrente das Guianas, que transporta as águas da plataforma externa e do talude na direção noroeste (Kuehl et al. 1986). O ciclo de marés e a pluviosidade fornecem nutrientes para a manutenção da fauna encontrada no sedimento lodoso e arenoso, favorecendo o crescimento de larvas e juvenis de peixes, crustáceos e moluscos (Isaac \& Braga 1999). Como efeito, a região possui uma alta produtividade biológica, sustentando uma elevada biomassa e uma complexa teia alimentar que influencia positivamente a atividade pesqueira local e de regiões adjacentes (Neiva \& Moura 1977, Wolff et al. 2000). O ambiente pelágico da plataforma, por outro lado, é considerado oligotrófico, sem áreas de ressurgências ou grandes arrecifes que poderiam influenciar o aumento da produtividade. A zona eufótica é pouco profunda e mantida por nutrientes provenientes da regeneração bentônica dos setores costeiros rasos e pela vazão dos rios e estuários, que acabam promovendo a fertilização das águas (Brasil 2006). A biota da costa norte do Brasil também é influenciada pela bacia do rio Orenoco, que possui aproximadamente $830.000 \mathrm{~km}^{2}$ (Lundberg et al. 1998). A descarga combinada de água doce do rio Amazonas e do rio Orenoco forma a "Pluma do Amazonas-Orenoco", que confere à região características oceanográficas particulares (Ekau \& Knoppers 1999, Brasil 2007), e representa uma importante barreira separando as províncias biogeográficas do Caribe e do Brasil (Luiz et al. 2012). Desta forma, as características bióticas e abióticas da costa norte do Brasil permitem o reconhecimento de uma ecorregião distinta no Atlântico ocidental (e.g., Sullivan Sealey \& Bustamante 1999, Spalding et al. 2007). A ictiofauna da costa norte do Brasil possui grande valor comercial e social. A atividade pesqueira é predominantemente artesanal, caracterizada pela utilização de barcos de madeira e operação manual dos aparelhos de pesca (Isaac-Nahum 2006, Bentes et al. 2012). A atuação da frota pesqueira restringe-se à área costeira e à plataforma interna, entre o limite norte do Brasil e o litoral do Maranhão (Isaac et al. 2008). A pesca na região baseia-se em artes de pesca variadas, como linhas de mão simples, espinhéis de fundo, redes de emalhe de grande porte, currais e covos, com destaque para aquelas de maior importância econômica, como redes de espera e tapagem (Espírito-Santo et al. 2005).

Apesar de sua relevância em termos biogeográficos, ecológicos e comerciais, a fauna de peixes da costa norte do Brasil é pouco conhecida, representando a região menos amostrada e estudada de toda ZEE brasileira. Neste artigo, apresentamos um panorama geral do conhecimento dos peixes estuarinos e marinhos da costa norte do Brasil. Além disso, procuramos identificar as principais razões pelas quais a ictiofauna da região é pouco conhecida, sugerindo medidas para que a situação seja revertida.

\section{Material e Métodos}

Os catálogos de 14 coleções ictiológicas brasileiras com livre acesso na internet foram consultados através dos sítios dos projetos Neodat (http://www.mnrj.ufrj.br/search.htm) e SpeciesLink (http:// www.splink.org.br/), com dados completos da Coleção de Peixes do Museu Paraense Emílio Goeldi (MPEG) e do Museu Nacional, Universidade Federal do Rio de Janeiro (MNRJ), obtidos diretamente com os curadores dessas coleções, totalizando 279.890 lotes. O número de famílias de peixes marinhos (Chondrichthyes e Teleostei), citados para as diferentes regiões da costa brasileira, foi estimado com base nos acervos da coleção de Peixes do Museu Paraense Emílio Goeldi (MPEG), do Museu Nacional, Universidade Federal do Rio de Janeiro (MNRJ), Museu de Ciências e Tecnologia da Pontifícia Universidade Católica do Rio Grande do Sul, Porto Alegre, Rio Grande do Sul (MCP) e do Museu de Zoologia da Universidade de São Paulo (MZUSP), que são as mais representativas em relação aos peixes marinhos e estuarinos brasileiros. A Figura 1 foi produzida no Quantum GIS (QGIS), com livre acesso em http://www.qgis.org.

\section{Resultados e Discussão}

A compilação mais completa e confiável sobre a ictiofauna marinha do Brasil, realizada por Menezes et al. (2003), apresenta um sumário das espécies com ocorrência documentada ou altamente provável na costa brasileira, totalizando 1.297 espécies válidas, das quais 925 são registradas ou potencialmente ocorrem na costa norte do Brasil. Das 200 famílias de peixes reconhecidas por Menezes et al. (2003), 146 supostamente são encontradas na costa norte brasileira. No entanto, a maioria absoluta dos registros atribuídos à região provém de inferências feitas a partir de espécies com registros mais ao norte, como na região das Guianas, ou no nordeste do Brasil. Dados de ocorrência, documentados a partir de exemplares coletados na costa norte do Brasil são escassos e refletem a ausência de material representativo em coleções científicas (Menezes et al. 2003).

O levantamento do acervo das coleções ictiológicas brasileiras mostra o desequilíbrio no esforço de inventariamento da fauna marinha nas diferentes regiões do Brasil. A diferença no conhecimento da biodiversidade marinha da região Norte brasileira é significativamente expressa pelo número de famílias de peixes marinhos e estuarinos representados em coleções zoológicas brasileiras, com 91 famílias atribuídas à região Nordeste, 109 famílias à região Sudeste, 117 famílias à região Sul, e apenas 48 famílias à região Norte (Tabela 1).

Em números absolutos, o acervo de peixes marinhos e estuarinos da região Norte representa apenas 3,9\% do total dos lotes catalogados em coleções brasileiras, contra $40 \%$ da região Sudeste e 39,9\% da região Sul, e os 16,2\% restantes da região Nordeste (Figura 2). Conseqüentemente, as espécies da região Norte, encontradas em coleções zoológicas, possuem baixa representatividade, mesmo aquelas abundantes e de fácil amostragem em zonas costeiras e estuarinas. Os bagres marinhos da família Ariidae são um bom exemplo desta situação (Marceniuk \& Menezes 2007). A família é representada por 176 lotes de 15 espécies na região Norte, contra 357 lotes de 10 espécies na região Sudeste e 628 lotes de 4 espécies na região Sul, com uma média de 11,7 lotes por espécie para a região Norte, 35,7 para a região Sudeste, e o expressivo número de 157 lotes por espécie para a região Sul. Condição similar é observada em outras famílias comercialmente importantes e com taxonomia complexa, como Centropomidae e Mugilidae.

O desequilíbrio no conhecimento da biota marinha do Brasil também é evidenciado pela literatura disponível para as diferentes regiões do país. Até o final do século XX, menos de 10\% dos mais de 100 estuários brasileiros haviam sido estudados, com o número de espécies de peixes citados variando entre 21 a 118 (Vazzoler et al. 1999). No mesmo período, existia uma clara diferença do esforço de inventariamento e estudo da biologia dos peixes das diferentes regiões do Brasil. O estuário Amazônico $\left(0^{\circ} \mathrm{S}-3^{\circ} \mathrm{S}\right)$ e a Lagoa dos Patos $\left(32^{\circ} \mathrm{S}\right)$, os dois principais sistemas estuarinos-lagunares brasileiros, 


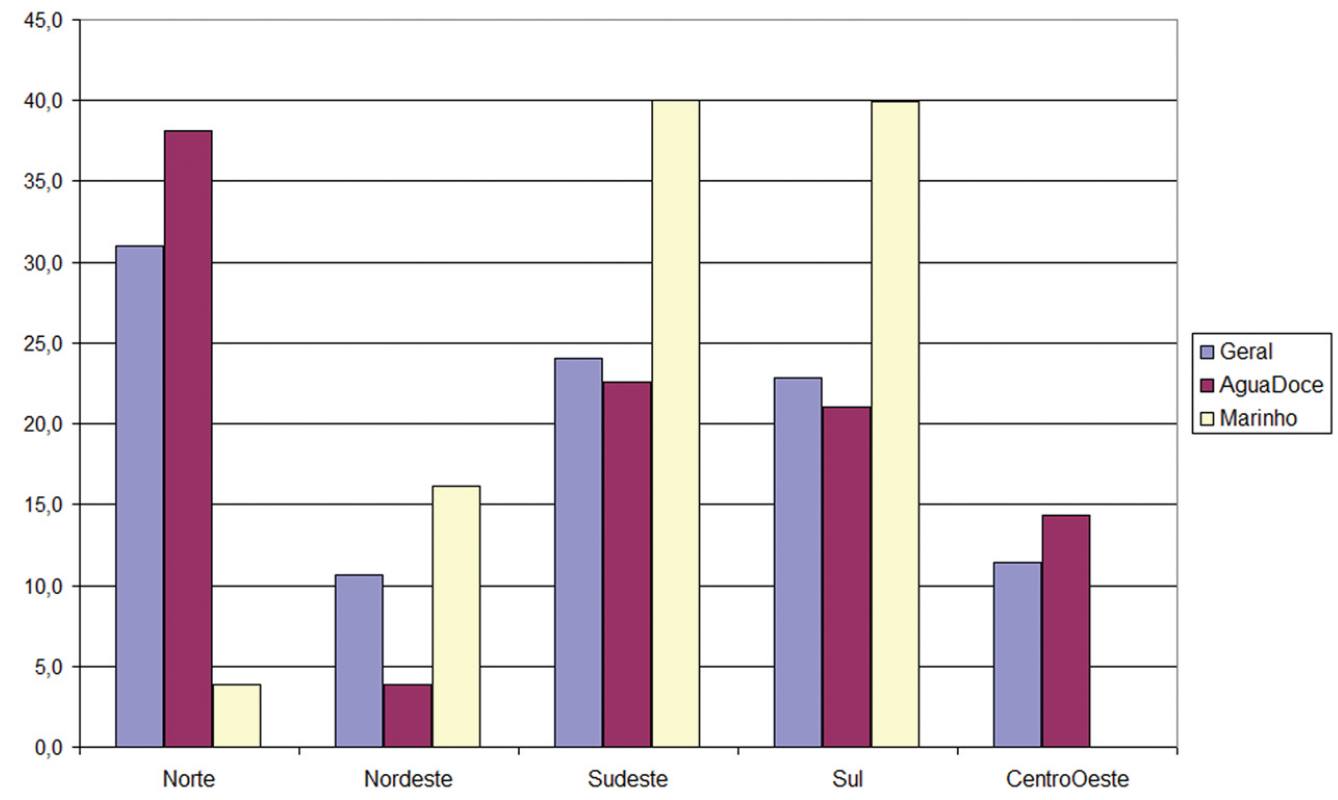

Figura 2. Valores relativos (em porcentagem) do número de lotes de peixes depositados em coleções zoológicas nas cinco regiões geopolíticas brasileiras. Em azul são indicados os valores totais, incluindo espécies de água doce e marinhas. Em vinho, apenas espécies de água doce são indicadas. Em amarelo, apenas espécies estuarinas e marinhas são indicadas.

Figure 2. Relative values (in percentages) of the number of fish lots deposited in zoological collections in the five Brazilian geopolitical regions. In blue, total value is indicated, including freshwater and marine species. In wine, only freshwater species is indicated. In yellow, only estuarine and marine species is indicated.

servem como exemplo do descompasso existente em relação ao conhecimento da biodiversidade estuarina das regiões Norte e Sul do Brasil. Até o início do século XXI havia sido publicado apenas um estudo sobre os peixes do estuário Amazônico e da plataforma continental adjacente (Barthem 1985), condição bastante diferente daquela observada para a Lagoa dos Patos, intensamente estudada em relação à composição da ictiofauna (ver Chao et al. 1985) e à biologia das espécies (ver Haimovici 1997).

Atualmente, praticamente inexistem publicações a respeito da composição de peixes marinhos-estuarinos da costa Norte do Brasil, e os poucos trabalhos disponíveis têm caráter local (Martins-Juras et al. 1987, Camargo \& Isaac 2001, Rocha \& Rosa 2001, EspíritoSanto et al. 2005, Feitoza et al. 2005), impedindo a realização de extrapolações regionais da biodiversidade. Na revisão da literatura sobre a ictiofauna estuarina do Brasil, realizada por Ribeiro (2007), é reconhecida a elevada riqueza de espécies estuarinas, com o registro 530 espécies pertencentes a 114 famílias. Contudo, Ribeiro (2007) concluiu que o conhecimento da biodiversidade estuarina brasileira é bastante heterogêneo, sendo as costas do Nordeste e do Sudeste detentoras da maior riqueza de espécies ( 279 spp., pertencentes a 83 e 81 famílias, respectivamente), e a região Sul com a menor riqueza (223 spp., incluídas em 72 famílias). Por sua vez, os estuários da região Amazônica apresentam uma riqueza intermediária (242 spp. e 75 famílias). O resultado reflete o parco conhecimento sobre a ictiofauna marinha da região Norte, existindo apenas 27 artigos publicados referentes às regiões Norte e Nordeste, incluindo estudos de biologia, ecologia e inventariamento, contra 74 trabalhos referentes à região Sudeste e 105 trabalhos referentes à região Sul (sensu Ribeiro 2007). Recentemente, prospecções realizadas pelo Programa REVIZEE propiciaram avanços no conhecimento da ictiofauna brasileira, especialmente nas regiões Sudeste-Sul (e.g. Figueiredo et al. 2002, Bernardes et al. 2005), Central (Rocha \& Costa 1999) e Nordeste (Lessa \& Nóbrega 2000, Coelho 2004, Vaske Junior et al. 2004). Os resultados disponíveis até o momento para costa Norte são em grande parte focalizados em poucas espécies de importância pesqueira (e.g. Asano-Filho et al. 2005, Frédou \& AsanoFilho 2006). Embora relevantes, esses estudos não representam uma adição significativa ao conhecimento da diversidade alfa de peixes da região.

$\mathrm{O}$ atual estado do conhecimento da costa norte brasileira também é reflexo do número reduzido de sistematas atuando na região Norte, e da distribuição desigual das coleções zoológicas no Brasil. Das 54 coleções de peixes conhecidas em território brasileiro, 35 estão situadas na região Sudeste-Sul e 12 estão na região Nordeste, enquanto apenas seis estão localizadas na região Norte. O número insuficiente de sistematas atuando de maneira ativa também representa um problema sério para o conhecimento da biodiversidade (UNESCO 1988, Gaston \& May 1992). Nesse sentido, a falta de sistematas trabalhando com organismos marinhos na costa norte é particularmente dramática (Couto et al. 2003, Amaral \& Jablonski 2005). Em levantamento recente sobre o panorama atual da sistemática no Brasil, foram identificados 542 pesquisadores que se autodenominam sistematas ou taxonomistas (Marques \& Lamas 2006). Destes, apenas $10,5 \%$ atuam na região Norte, contra um número significativamente maior atuando na região Sudeste $(51,7 \%)$. Em termos do número de publicações em sistemática produzidas no Brasil, entre 2000 e 2005, a região Norte ocupa a última colocação, contribuindo com apenas 4,5\% do total de publicações, contra 70,6\% da região Sudeste. Marques \& Lamas (2006) também ressaltaram que essa situação é mais extrema em relação ao conhecimento da fauna marinha, onde a amostragem é praticamente nula, sendo toda a fauna bentônica da região pouco conhecida (Kempf et al. 1967, Kempf 1970, Aller \& Aller 1986). O próprio Museu Paraense Emilio Goeldi (MPEG), detentor da maior coleção zoológica da região Norte do Brasil, possui uma pequena amostragem da biodiversidade marinha e estuarina, refletindo a ausência histórica de pesquisadores vinculados à instituição, com foco no ambiente marinho. A situação é preocupante, pois enquanto uma parte considerável da biodiversidade permanece desconhecida, os recursos naturais vêm sendo explorados em ritmo cada vez mais intenso. 
Peixes marinhos e estuarinos da costa norte do Brasil

Tabela 1. Lista das 143 famílias de peixes marinhos e estuarinos (Chondrichthyes e Teleostei) com lotes depositados em coleções zoológicas brasileiras. A ausência de registros de famílias em coleções zoológicas nas quatro regiões geopolíticas do Brasil é indicada por "X". Abreviaturas: região Norte (N), região Nordeste $(\mathrm{Nd})$, região Sudeste $(\mathrm{Sd})$, e região Sul (S); “Total” refere-se ao número de famílias registradas em cada região.

Table 1. List of 143 families of marine and estuarine fishes (Chondrichthyes and Teleostei) with lots deposited in Brazilian zoological collections. Absence of records of families in zoological collections of the four geopolitical regions of Brazil is indicated by "X". Abbreviations: North (N), Northeast (Nd), Southeast (Sd) and Southern (S). "Total" refers to the number of families recorded in each region.

\begin{tabular}{|c|c|c|c|c|c|c|c|c|c|c|c|c|c|c|}
\hline Família & $\mathbf{N}$ & Nd & Sd & $\mathbf{S}$ & Família & $\mathbf{N}$ & Nd & Sd & $\mathbf{S}$ & Família & $\mathbf{N}$ & Nd & Sd & $\mathbf{S}$ \\
\hline Acanthuridae & & & & & Ephippidae & & & & & Percophidae & $\mathrm{x}$ & $\mathrm{x}$ & & \\
\hline Achiridae & & & & & Exocoetidae & $\mathrm{x}$ & & & & Peristediidae & $\mathrm{x}$ & & & \\
\hline Acropomatidae & $\mathrm{x}$ & $\mathrm{x}$ & $\mathrm{x}$ & & Fistulariidae & $\mathrm{x}$ & & & & Phosichthyidae & $\mathrm{x}$ & $\mathrm{x}$ & $\mathrm{x}$ & \\
\hline Albulidae & $\mathrm{x}$ & & & $\mathrm{x}$ & Gempylidae & $\mathrm{x}$ & $\mathrm{x}$ & & & Phycidae & $\mathrm{x}$ & $\mathrm{x}$ & & \\
\hline Antennariidae & $\mathrm{x}$ & & & & Gerreidae & & & & & Pinguipedidae & $\mathrm{x}$ & $\mathrm{x}$ & & \\
\hline Apogonidae & $\mathrm{x}$ & & & & Ginglymostomatidae & $\mathrm{x}$ & $\mathrm{x}$ & & $\mathrm{x}$ & Pleuronectidae & $\mathrm{x}$ & $\mathrm{x}$ & & \\
\hline Argentinidae & & $\mathrm{x}$ & $\mathrm{x}$ & & Gobiesocidae & $\mathrm{x}$ & & & & Polymixiidae & & & & \\
\hline Ariidae & & & & & Gobiidae & & & & & Polyprionidae & $\mathrm{x}$ & $\mathrm{x}$ & $\mathrm{x}$ & \\
\hline Ariommatidae & $\mathrm{x}$ & $\mathrm{x}$ & & & Grammatidae & $\mathrm{x}$ & & & $\mathrm{x}$ & Pomacanthidae & $\mathrm{x}$ & & & \\
\hline Atherinidae & & & & & Grammicolepididae & $\mathrm{x}$ & $\mathrm{x}$ & $\mathrm{x}$ & & Pomacentridae & $\mathrm{x}$ & & & \\
\hline Atherinopsidae & & & & & Gymnuridae & $\mathrm{x}$ & & & & Pomatomidae & $\mathrm{x}$ & & & \\
\hline Aulostomidae & $\mathrm{x}$ & & & $\mathrm{x}$ & Haemulidae & & & & & Priacanthidae & $\mathrm{x}$ & & & \\
\hline Balistidae & $\mathrm{x}$ & & & & Hemiodontidae & $\mathrm{x}$ & & & & Pristidae & & $\mathrm{x}$ & $\mathrm{x}$ & $\mathrm{x}$ \\
\hline Batrachoididae & & & & & Hemiramphidae & $\mathrm{x}$ & & & & Pristigasteridae & & $\mathrm{x}$ & $\mathrm{x}$ & $\mathrm{x}$ \\
\hline Belonidae & & & & & Holocentridae & $\mathrm{x}$ & & & & Rachycentridae & $\mathrm{x}$ & $\mathrm{x}$ & & $\mathrm{x}$ \\
\hline Berycidae & $\mathrm{x}$ & $\mathrm{x}$ & $\mathrm{x}$ & & Kyphosidae & $\mathrm{x}$ & & & & Rajidae & $\mathrm{x}$ & & & \\
\hline Blenniidae & & & & & Labridae & $\mathrm{x}$ & & & & Rhinobatidae & $\mathrm{x}$ & & & \\
\hline Bothidae & & & & & Labrisomidae & $\mathrm{x}$ & & & & Scaridae & $\mathrm{x}$ & & & \\
\hline Bramidae & $\mathrm{x}$ & $\mathrm{x}$ & $\mathrm{x}$ & & Lobotidae & & & & & Sciaenidae & & & & \\
\hline Bregmacerotidae & $\mathrm{x}$ & $\mathrm{x}$ & $\mathrm{x}$ & & Lophiidae & $\mathrm{x}$ & $\mathrm{x}$ & $\mathrm{x}$ & & Scomberesocidae & $\mathrm{x}$ & $\mathrm{x}$ & $\mathrm{x}$ & \\
\hline Bythitidae & $\mathrm{x}$ & & $\mathrm{x}$ & $\mathrm{x}$ & Lutjanidae & & & & & Scombridae & & & & \\
\hline Callionymidae & $\mathrm{x}$ & & & & Macroramphosidae & $\mathrm{x}$ & $\mathrm{x}$ & $\mathrm{x}$ & & Scorpaenidae & $\mathrm{x}$ & & & \\
\hline Callorhynchidae & $\mathrm{x}$ & $\mathrm{x}$ & & & Macrouridae & $\mathrm{x}$ & $\mathrm{x}$ & $\mathrm{x}$ & & Scyliorhinidae & $\mathrm{x}$ & $\mathrm{x}$ & & \\
\hline Caproidae & $\mathrm{x}$ & & & & Malacanthidae & $\mathrm{x}$ & & & & Serranidae & & & & \\
\hline Carangidae & & & & & Megalopidae & & & $\mathrm{x}$ & $\mathrm{x}$ & Sparidae & $\mathrm{x}$ & & & \\
\hline Carapidae & $\mathrm{x}$ & & $\mathrm{x}$ & & Merlucciidae & $\mathrm{x}$ & $\mathrm{x}$ & & & Sphyraenidae & & & & \\
\hline Carcharhinidae & & & & & Microdesmidae & $\mathrm{x}$ & & & $\mathrm{x}$ & Sphyrnidae & & $\mathrm{x}$ & $\mathrm{x}$ & $\mathrm{x}$ \\
\hline Centrolophidae & & & & & Mobulidae & $\mathrm{x}$ & $\mathrm{x}$ & & $\mathrm{x}$ & Squalidae & $\mathrm{x}$ & $\mathrm{x}$ & & \\
\hline Chaenopsidae & $\mathrm{x}$ & & & & Monacanthidae & $\mathrm{x}$ & & & & Squatinidae & $\mathrm{x}$ & $\mathrm{x}$ & & \\
\hline Chaetodontidae & $\mathrm{x}$ & & & & Moringuidae & $\mathrm{x}$ & & $\mathrm{x}$ & $\mathrm{x}$ & Sternoptychidae & $\mathrm{x}$ & $\mathrm{x}$ & $\mathrm{x}$ & \\
\hline Cheilodactylidae & $\mathrm{x}$ & $\mathrm{x}$ & & & Mugilidae & & & & & Stomiidae & $\mathrm{x}$ & $\mathrm{x}$ & $\mathrm{x}$ & \\
\hline Chlopsidae & $\mathrm{x}$ & & $\mathrm{x}$ & $\mathrm{x}$ & Mullidae & & & & & Stromateidae & & & & \\
\hline Chlorophthalmidae & $\mathrm{x}$ & $\mathrm{x}$ & $\mathrm{x}$ & & Muraenesocidae & $\mathrm{x}$ & & $\mathrm{x}$ & $\mathrm{x}$ & Synaphobranchidae & $\mathrm{x}$ & $\mathrm{x}$ & $\mathrm{x}$ & \\
\hline Cirrhitidae & $\mathrm{x}$ & & & $\mathrm{x}$ & Muraenidae & & & & & Syngnathidae & $\mathrm{x}$ & & & \\
\hline Clinidae & $\mathrm{x}$ & $\mathrm{x}$ & & & Myctophidae & $\mathrm{x}$ & $\mathrm{x}$ & $\mathrm{x}$ & & Synodontidae & $\mathrm{x}$ & & & \\
\hline Clupeidae & & & & & Myliobatidae & & $\mathrm{x}$ & & & Tetraodontidae & & & & \\
\hline Congridae & $\mathrm{x}$ & & & & Narkidae & & & & & Torpedinidae & & $\mathrm{x}$ & $\mathrm{x}$ & $\mathrm{x}$ \\
\hline Coryphaenidae & $\mathrm{x}$ & $\mathrm{x}$ & & & Neoscopelidae & $\mathrm{x}$ & $\mathrm{x}$ & $\mathrm{x}$ & & Trachichthyidae & $\mathrm{x}$ & $\mathrm{x}$ & $\mathrm{x}$ & \\
\hline Cynoglossidae & & & & & Nettastomatidae & $\mathrm{x}$ & $\mathrm{x}$ & $\mathrm{x}$ & & Trachinidae & $\mathrm{x}$ & $\mathrm{x}$ & & $\mathrm{x}$ \\
\hline Dactylopteridae & $\mathrm{x}$ & & & & Nomeidae & $\mathrm{x}$ & $\mathrm{x}$ & & $\mathrm{x}$ & Triakidae & $\mathrm{x}$ & $\mathrm{x}$ & & \\
\hline Dactyloscopidae & $\mathrm{x}$ & & & $\mathrm{x}$ & Odontaspididae & $\mathrm{x}$ & $\mathrm{x}$ & & & Trichiuridae & & & & \\
\hline Dasyatidae & & & & & Ogcocephalidae & & & & & Triglidae & & & & \\
\hline Diodontidae & & & & & Ophichthidae & $\mathrm{x}$ & & & & Tripterygiidae & $\mathrm{x}$ & & & $\mathrm{x}$ \\
\hline Echeneidae & & & & $\mathrm{x}$ & Ophidiidae & $\mathrm{x}$ & & & & Uranoscopidae & $\mathrm{x}$ & $\mathrm{x}$ & & \\
\hline Eleotridae & & & & & Opistognathidae & $\mathrm{x}$ & & & & Xiphiidae & $\mathrm{x}$ & $\mathrm{x}$ & & $\mathrm{x}$ \\
\hline Elopidae & & & & & Ostraciidae & $\mathrm{x}$ & & & & Zeidae & $\mathrm{x}$ & $\mathrm{x}$ & $\mathrm{x}$ & \\
\hline Engraulidae & & & & & Paralichthyidae & & & & & Total & 48 & 91 & 109 & 117 \\
\hline
\end{tabular}




\section{Perspectivas do desenvolvimento de estudos em sistemática e taxonomia de peixes marinhos e estuarinos}

O conhecimento da diversidade biológica, realizado através da descoberta e descrição de espécies (taxonomia alfa), é relativamente completo em algumas regiões do mundo, especialmente no que diz respeito a famílias com maior interesse comercial. Entretanto, lacunas importantes de conhecimento ainda existem. Nos últimos anos, novas espécies de peixes marinhos têm sido descritas, a uma taxa de cerca de 100-150 por ano (Eschmeyer et al. 2010). Segundo Mora et al. (2008), com base em registros do banco de dados do "Ocean Biogeographical Information System (OBIS)" (http://www. obis.org), cerca de $21 \%$ do total de espécies de peixes marinhos, aproximadamente 3.200 espécies, precisam ser descritas. Diversos autores consideram essas estimativas ilusórias, e reconhecem um número muito maior de espécies de peixes marinhos nesta situação. Eschmeyer et al. (2010) estimaram que o número de peixes marinhos não descritos estaria ao redor de 5.000, o que equivale a duas vezes o número de espécies descritas nos últimos 19 anos. Os autores também argumentam que áreas menos amostradas, ou que possuem pouco material representativo em coleções zoológicas, guardam um maior número de espécies a serem descritas. Por exemplo, apenas duas espécies marinhas foram descritas no Canadá nos últimos 40 anos, contra 38 espécies marinhas descritas nos últimos nove anos em águas brasileiras, número inferior somente ao da Austrália (240 spp.), Japão (90 spp.), e Taiwan (43 spp.) (Eschmeyer et al. 2010).

A expectativa de Eschmeyer et al. (2010) vem sendo confirmada nos últimos anos a partir de coletas realizadas principalmente nas regiões Nordeste e Sudeste do Brasil, que possibilitaram a descrição de novas espécies pertencentes a grupos filogeneticamente distintos, como Myxiniformes, Chondrichthyes, Tetraodontiformes e Perciformes. A maior parte das espécies descritas ocorre em ambientes recifais (e.g., Moura 1995, Sazima et al. 1997, 1998, 2008, 2009, Gasparini et al. 1999, Heiser et al. 2000, Moura et al. 2001, Pinheiro et al. 2010), ou de águas profundas (e.g., Mincarone 2000, 2001, Soto 2001a,b, Gomes \& Paragó 2001, Soto \& Vooren 2004, Carvalho et al. 2005, Anderson \& Mincarone 2006, Melo 2007, Mincarone \& Anderson 2008, Nielsen 2009), ambientes historicamente mal amostrados. Além disso, diversos trabalhos têm registrado novas ocorrências de espécies, até então, não assinaladas no Atlântico Sul (e.g., Barreiros et al. 2004, Lima \& Mincarone 2004, Mincarone \& Sampaio 2004, Feitoza et al. 2005, Mincarone \& Smith 2005, Mincarone et al. 2008, Carvalho-Filho et al. 2009, 2010a, b, 2011, 2012, Franco et al. 2009, Melo et al. 2009, 2010, Lima et al. 2011). O exame de séries maiores de exemplares e ferramentas moleculares também vêm contribuindo para o melhor conhecimento da biodiversidade marinha com a revalidação de espécies nominais sinonimizadas (e.g. Marceniuk 2005, 2007, Carvalho-Filho et al. 2010c)

Da mesma forma, coletas em áreas marinhas da costa norte brasileira podem revelar uma fauna desconhecida. Carvalho-Filho (1999), por exemplo, indicou a possível ocorrência das sardinhas de pequeno porte Etrumeus teres (De Kay 1842) e Jenkinsia lamprotaenia (Gosse 1851) na região. Essas espécies fazem parte de Dussumieriinae (Clupeidae), um grupo de sardinhas altamente modificado em termos morfológicos, ainda muito pouco conhecido filogenética e biologicamente (Di Dario 2005). A confirmação da ocorrência destas espécies no Brasil representaria uma adição interessante ao patrimônio biológico brasileiro. O mesmo certamente se aplica a diversos grupos de peixes marinhos, que são mais comuns na porção norte da América do Sul e Caribe e que não possuem registros no Brasil.
A pesquisa direcionada ao melhor conhecimento da ictiofauna da costa norte do Brasil também é importante para o monitoramento de espécies invasoras no Atlântico, como o peixe leão, Pterois volitans (Linnaeus 1758) e P. miles (Bennett 1828), da família Scorpaenidae que são naturais do Indo-Pacífico. Os primeiros registros do peixe leão no Atlântico Central ocidental foram feitos em meados da década de 1980, na Flórida (Schofield 2009). O primeiro registro de $P$. volitans na América do Sul ocorreu em 2008, na Colômbia (Schofield 2009, González et al. 2009). Atualmente, o peixe leão é encontrado nas Bahamas e em outras regiões do Caribe, incluindo as Ilhas Cayman, Cuba, Jamaica, República Dominicana, Porto Rico, Honduras Costa Rica, Haití, México e Panamá (Schofield 2009) e também na Venezuela (Lasso-Alcalá \& Posada 2010). Ainda não existem registros confirmados de $P$. volitans na Guianas, Suriname e Brasil. Apesar disso, o ritmo de expansão, resiliência, e capacidade de colonização de novos ambientes, sugerem que a espécie poderá expandir sua área de distribuição no Atlântico Sul ocidental (Luiz et al. 2013). O reconhecimento precoce da presença da espécie na costa norte do Brasil pode representar uma chance para o desenvolvimento de medidas de controle, minimizando possíveis impactos aos ambientes recifais brasileiros.

\section{Conclusão}

Os resultados apresentados indicam uma diferença marcante no esforço de inventariamento dos peixes marinhos nas diferentes regiões do pais. Na costa norte, essa diferença é reflexo do pequeno número de pesquisadores trabalhando com a sistemática, biologia e ecologia de peixes marinhos. $\mathrm{O}$ conhecimento insatisfatório sobre a diversidade das espécies marinhas e estuarinas da costa norte do Brasil implica na incapacidade de desenvolver políticas públicas adequadas de conservação, com consequências deletérias não apenas à manutenção da biodiversidade, mas também para à utilização racional dos recursos naturais da região. O Brasil é signatário da Convenção da Diversidade Biológica. Nesse sentido, o inventariamento e a disponibilização do conhecimento sobre a biodiversidade dos peixes marinhos são passos importantes para o cumprimento de compromissos internacionais assumidos pelo Brasil durante a Conferência das Nações Unidas sobre o Meio Ambiente e Desenvolvimento, realizada em junho de 1992 (Brasil 2002).

O melhor conhecimento da ictiofauna marinha da costa norte do Brasil passa pelo investimento em projetos de pesquisa nas áreas de sistemática, taxonomia e biogeografia. Além disso, as coleções científicas localizadas na região Norte precisam receber maior estímulo para o trabalho de inventariamento e formação de sistematas especializados em grupos marinhos, possibilitando o avanço na compreensão da biodiversidade de uma das regiões cientificamente mais carentes do Brasil. Outra ação importante, que pode trazer um aumento significativo na compreensão da ictiofauna marinha da região é a criação de bancos de DNA associados às coleções científicas da região, que poderão ser utilizados no esclarecimento de diversas questões relacionadas à biologia da conservação, como a identificação de espécies ou populações isoladas (Nash et al. 1998, Walpole et al. 2001), o desenvolvimento de estratégias de manejo (Geyer et al. 1993, Miller 1995) e a determinação do estado de conservação com base em parâmetros de estruturação da diversidade genética, bem como o desenvolvimento de análises filogenéticas e filogeográficas (O’Brien et al. 1985, Avise et al. 1987, 1995). Estudos espaciais e temporais de espécies ou populações utilizando marcadores moleculares também podem melhorar nosso conhecimento sobre aspectos da biogeografia e evolução da fauna brasileira, revelando relações de ancestralidade comum, possíveis rotas de migração e padrões de dispersão de espécies (Excoffier et al. 
1992, Avise et al. 1995, Culver et al. 2000). Esse conhecimento é essencial, por exemplo, para a definição de áreas prioritárias para conservação e manejo de espécies com importância comercial para pesca (Avise et al. 1987, 1995, Walpole et al. 2001).

\section{Agradecimentos}

Os autores agradecem a Marcelo R. Britto (MNRJ), pela disponibilização dos dados da Coleção de Peixes do Museu Nacional, Universidade Federal do Rio de Janeiro e pelo incentivo à confecção do manuscrito, e a Flávio C. T. Lima (UNICAMP), Naércio A. Menezes (MZUSP), Ierecê Maria de Lucena Rosa (UFPB), e Michael Maia Mincarone (UFRJ), pelos comentários e sugestões que melhoraram substancialmente o texto final. O primeiro autor agradece ao CNPq e a FAPESPA pelas bolsas de pesquisa PDS e DCR (processo 151731/2010-1 e 350790/2012-4). WBW agradece ao CNPq pela bolsa de produtividade em pesquisa (processo 304754/2011-0), e FDD pelo apoio financeiro da FAPERJ e CNPq.

\section{Referências Bibliográficas}

ALBERONI, A.A.L., JECK, I.K., TORRES, L.C. \& GORINI, M.A. 2007. A determinação da base do talude na região do cone do Amazonas. In Congresso Internacional da Sociedade Brasileira de Geofísica. Rio de Janeiro.

ALLER, J.Y. \& ALLER, R.C. 1986. Evidence for localized enhancement of biological associated with tube and burrow structures in deep-sea sediments at the HEEBLE site, western North Atlantic. Deep-Sea Res. 33(6):755-790. http://dx.doi.org/10.1016/0198-0149(86)90088-9

AMARAL, A.C.Z. \& JABLONSKI, S. 2005. Conservação da biodiversidade marinha e costeira do Brasil. Megadiversidade 1(1):43-51.

ANDERSON, M.E. \& MINCARONE, M.M. 2006. Studies on the Zoarcidae (Teleostei: Perciformes) of the southern hemisphere. IX. A new species of Pachycara from the southwestern Atlantic. Zootaxa 1177:21-26.

ASANO-FILHO, M., HOLANDA, F.C.A.F, SANTOS, F.J.S. \& CAVALCANTE JUNIOR, T.S. 2005. Recursos pesqueiros de grandes profundidades na costa Norte do Brasil. IBAMA, Brasília, p.1-82.

AVISE, J.C., ARNOLD, J., BALL, R.M., BERMINGHAM, E., LAMB, T., NEIGEL, J.E., REEB, C.A. \& SAUNDERS, N.C. 1987. Intraspecific phylogeography: The mitochondrial DNA bridge between Population genetics and systematics. Annu. Rev. Ecol. Syst. 18:489-522.

AVISE, J.C., HAIG, S.M., RYDER, O.A., LYNCH, M. \& GEYER, C.J. 1995. Descriptive genetic studies: applications in population management and conservation biology. In Population management for survival and recovery (J.D. Ballou \& T.J. Fose, ed.). Columbia University Press, New York, p.183-244.

BARREIROS, J.P., MACHADO, L., HOSTIM-SILVA, M., SAZIMA, I. \& HEEMSTRA, P.C. 2004. First record of Polyprion oxygeneios (Perciformes: Polyprionidae) for the south-west Atlantic and a northernmost range extension. J. Fish Biol. 64:1439-1441. http://dx.doi. org/10.1111/j.0022-1112.2004.00396.x

BARTHEM, R.B. 1985. Ocorrência, distribuição e biologia dos peixes da Baía de Marajó, estuário amazônico. Bol. Mus. Para. Emílio Goeldi ser. Zool. 2(1):49-69.

BENTES, B., ISAAC, V.J., ESPÍRITO-SANTO, R.V., FRÉDOU, T. ALMEIDA, M.C., MOURÃO, K.R.M. \& FRÉDOU, F.L. 2012. Multidisciplinary approach to identification of fishery production systems on the northern coast of Brazil. Biota Neotrop. 12(1): http:// www.biotaneotropica.org.br/v12n1/en/abstract?article+bn00612012012

BERNARDES, R.A., MELLO, G.P.M.B. \& CERGOLE, M.C. 2005. Helicolenus lahillei (Norman, 1937). In Análise das Principais Pescarias Comerciais da Região Sudeste-Sul do Brasil: Dinâmica Populacional das Espécies em Explotação (M.C. Cergole, A.O. Ávila-da-silva \& C.L.D.B. Rossi-Wongtschowski, eds.). Instituto Oceanográfico, São Paulo, p.1-176. Série Documentos Revizee- Score Sul.
BRASIL. Ministério do Meio Ambiente - MMA. 2002. Avaliação e ações prioritárias para a conservação da biodiversidade das zonas costeira e marinha. Biodiversidade Brasileira: Avaliação e Identificação de Áreas Prioritárias para Conservação, Utilização Sustentável e Repartição de Benefícios da Biodiversidade Brasileira. Série Biodiversidade, v. 5, p.1-404.

BRASIL. Ministério do Meio Ambiente - MMA. 2006. Programa REVIZEE: avaliação do potencial sustentável de recursos vivos na zona econômica exclusiva. Relatório Executivo, p.1-303.

BRASIL. Ministério do Meio Ambiente - MMA. 2007. Áreas Prioritárias para a, Conservação Uso Sustentável e Repartição de Benefícios da Biodiversidade Brasileira: Atualização - Portaria MMA n. 09, de 23 de janeiro de 2007. Série Biodiversidade 31, p.1-300.

CARVALHO, M.R., GOMES, U.L. \& GADIG, O.B.F. 2005. Description of a new species of skate of the genus Malacoraja Stehmann, 1970: the first species from the southwestern Atlantic Ocean, with notes on generic monophyly and composition (Chondrichthyes: Rajidae). Neotrop. Ichthyol. 2:238-259.

CARVALHO-FILHO, A. 1999. Peixes: costa brasileira. Editora Melro, São Paulo, p.1-320.

CARVALHO-FILHO, A., MARCOVALDI, G., SAMPAIO, C.L.S., PAIVA, M.I.G. \& DUARTE, L.A.G. 2009. First report of rare pomfrets (Teleostei: Bramidae) from Brazilian waters, with a key to western Atlantic species. Zootaxa 2290:1-26.

CARVALHO-FILHO, A., MARCOVALDI, G., SAMPAIO, C.L.S., PAIVA, M.I.G. \& DUARTE, L.A.G. 2010a. First report of Aulopus (Teleostei: Aulopidae) from southwestern Atlantic, with a review of records and a key to western Atlantic Aulopoidei species. Zootaxa 2628:27-42.

CARVALHO-FILHO, A., MARCOVALDI, G., SAMPAIO, C.L.S., PAIVA, M.I.G. \& DUARTE, L.A.G. 2010b. Two new records of uncommon deepsea perciform fishes from the southwestern Atlantic. Zootaxa 2694:59-68.

CARVALHO-FILHO, A., SANTOS, S., SAMPAIO, I. 2010c. Macrodon atricauda (Gunther, 1880) (Perciformes: Sciaenidae), a valid species from the southwestern Atlantic, with comments on its conservation. Zootaxa 2519:48-58

CARVALHO-FILHO, A., MARCOVALDI, G., SAMPAIO, C.L.S. \& PAIVA, M.I.G. 2011. First report of Macruronus novaezelandiae (Gadiformes: Merluccidae: Macruroninae) from Atlantic tropical waters. Marine Biodiversity Records 4:3. http://dx.doi.org/10.1017/S1755267211000431

CARVALHO-FILHO, A., MARCOVALDI, G., ALMEIDA RIBEIRO, F., PAIVA, M.I.G. \& SAMPAIO, C.L.S. 2012. New records of Grammicolepis brachiusculus Poey, 1873 (Zeiformes: Grammicolepididae) in Brazilian waters, with a key to western Atlantic species of Grammicolepididae. Check List 8(4):626-629.

CAMARGO, M. \& ISAAC, V. 2001. Os estuarinos da região norte do Brasil: espécies e considerações sobre sua distribuição geográfica. Bol. Mus. Para. Emílio Goeldi, sér. Zool. 17(2):135-157.

CHAO, L.N., PEREIRA, L.E. \& VIEIRA, J.P. 1985. Estuarine fish community of the dos Patos Lagoon, Brazil. A baseline study. In: Fish community ecology in estuaries and coastal lagoons: towards an ecosystem integration (A. Yáñez-Arancibia, ed.). DR(R) UNAM, México, p.429-450.

COELHO, P.A.F. 2004. Análise do macrobentos da plataforma continental externa e bancos oceânicos do nordeste do Brasil. Relatório final no âmbito do Programa REVIZEE, p.1-81.

COSTA, E.A. \& FIGUEIREDO JUNIOR, A.G. 1998. Echo-character and sedimentary processes on the Amazon continental shelf. An. Acad. Bras. Ciênc. 70(2):187-200.

COUTO, E.C.G., DA SILVEIRA, F.L. \& ROCHA, G.R.A. 2003. Marine biodiversity in Brazil: the current status/ Biodiversidad marina en brasil: estado actual del conocimento. Gayana 67(2):327-340.

CULVER M., JOHNSON, W.E., PECON-SLATTERY, J. \& O'BRIEN, S.J. 2000. Genomic ancestry of the American puma (Puma concolor). J. Hered. 91:186-197. http://dx.doi.org/10.1093/jhered/91.3.186

DI DARIO, F. 2005. Relações filogenéticas entre os grandes grupos de Clupeomorpha e suas possíveis relações com Ostariophysi (Actinopterygii, Teleostei). Tese de Doutorado, Instituto de Biociências, Universidade de São Paulo, São Paulo, p.1-640.

DITTMAR, T. 1999. Outwelling of Organic Matter and Nutrients from a mangrove in North Brazil: Evidence from Organic Tracers and Flux Measurements. ZMT contribution n. 5, p.1-229. 
EKAU, W. \& KNOPPERS, B. 1999. An introduction to the pelagic system of the North-East and East Brazilian shelf. Arch. Fish. Mar. Res. 47(23):113-132.

EL-ROBRINI, M., FARIA, L.E.C., TORRES, A.M., SOUZA, F.P.W.M. \& SILVA, M.S. 1992. Deposição e assoreamento das Rias do Nordeste do Estado do Pará (Brasil). In: Congresso Brasileiro de Geologia. São Paulo. Resumo, p.79-80.

ESCHMEYER, W.N., FRICKE, R., FONG, J.D. \& POLACK, D.A. 2010. Marine fish diversity: history of knowledge and discovery (Pisces). Zootaxa 2525:19-50.

ESPÍRITO-SANTO, R.V., ISAAC, V.J., SILVA, L.M.A., MARTINELLI, J.M., HIGUCHI, H. \& PAUL, U.S. 2005. Peixes e camarões do litoral bragantino, Pará, Brasil. Belém: Programa Madam, Manejo e Dinâmica de Áreas de Manguezais. 1, p.1-268.

EXCOFFIER, L., SMOUSE, P.E. \& QUATTRO, J.M. 1992 Analysis of molecular variance inferred from metric distances among DNA haplotypes: Application to human mitochondrial DNA data. Genetics 131:479-491.

FEITOZA, B.M., ROSA, R.S. \& ROCHA, L.A. 2005. Ecology and biogeography of deep-reef fishes in northeastern Brazil. Bull. Mar. Sci. 76(3):725-742.

FIGUEIREDO J.L., SANTOS, A.P., YAMAGUTI, N., BERNARDES, R.A. \& ROSSIWONGTSCHOWSKI, C.LD.B. 2002. Peixes da Zona Econômica Exclusiva da Região Sudeste-Sul do Brasil : Levantamento com Rede de Meia Água. EDUSP, São Paulo, p.1-244.

FRANCO, M.A., BRAGA, A.C., NUNAN, G.W. \& COSTA, P.A. 2009. Fishes of the family Ipnopidae (Teleostei: Aulopiformes) collected on the Brazilian continental slope between 11 degrees and 23 degrees S. J Fish. Biol. 75(4):797-815. http://dx.doi.org/10.1111/j.1095-8649.2009.02324.x

FRÉDOU, F.L. \& ASANO-FILHO, M. 2006. Recursos pesqueiros da região Norte. Programa REVIZEE: Avaliação do Potencial Sustentável de Recursos Vivos na Zona Econômica Exclusiva. Relatório Executivo, MMA, p.1-300.

GASPARINI, J.L., MOURA, R.L. \& SAZIMA, I. 1999. Stegastes trindadensis n. sp. (Pisces: Pomacentridae), a new damselfish from Trinidade Island, off Brazil. Bol. Mus. Biol. Mello Leitão 10:3-11.

GASTON, K.J. \& MAY, R.M. 1992. Taxonomy of taxonomists. Nature 356:281-283. http://dx.doi.org/10.1038/356281a0

GEYER, C.J., RYDER, O.A., CHEMNICK, L.G. \& THOMPSON, E.A. 1993. Analysis of relatedness in the California condors from DNA fingerprints. Mol. Biol. Evol. 10:571-589.

GOMES, U.L. \& PARAGÓ, C. 2001. Espécie nova de Rajídeo (Chondrichthyes, Rajiformes) do Atlântico sul occidental. Bol. Mus. Nac., NS, Zool. 448:1-10.

GONZÁLEZ, J., GRIJALBA-BENDECK, M., ACERO P. A. \& BETANCUR, R. 2009. The invasive red lionfish, Pterois volitans (Linnaeus 1758), in the southwestern Caribbean Sea. Aquatic Invasions 4(3): 507-510.

HAIMOVICI, M. 1997. Recursos pesqueiros demersais da região sul. FEMAR, Rio de Janeiro, p.1-80.

HEISER, J.B., MOURA, R.L. \& ROBERTSON, D.R. 2000. Two new species of creole wrasse (Labridae: Clepticus) from opposite sides of the Atlantic. Aqua 4(2):67-76.

ISAAC, V.J.E \& BARTHEM, R.B. 1995. Os recursos pesqueiros da Amazônia brasileira. Bol. Mus. Para. Emílio Goeldi, ser. Antropo. 11(2):295-339.

ISAAC, V.J. \& BRAGA, T.M.P. 1999. Rejeição de pescado nas pescarias da região Norte do Brasil. Fortaleza, p.39-54.

ISAAC, V.J., ESPIRITO SANTO, R.V. \& NUNES, J.L.G. 2008. A estatística pesqueira no litoral do Pará: resultados divergentes. Panamjas 3(3):205213.

ISAAC-NAHUM, V.J. 2006. Explotação e manejo dos recursos pesqueiros do litoral Amazônico: um desafio para o futuro. Cienc. Cul. 58(3):33-36.

KEMPF, M., COUTINHO, P.N. \& MORAIS, J.O. 1967. Plataforma continental do Norte e Nordeste do Brasil I. Nota preliminar sobre a natureza do fundo. Trabalhos Oceanográficos da Universidade Federal de Pernambuco, v.9-11, p.9-26.

KEMPF, M. 1970. Notes on the benthic bionomy of NE Brazilian shelfs. Mar. Biol. 5(3):213-214. http://dx.doi.org/10.1007/BF00346909
KUEHL, S.A., DEMASTER, D.J. \& NITTROUER, C.A. 1986. Nature of sediment accumulation on the Amazon continental shelf. Cont. Shelf Res. 6:209-225. http://dx.doi.org/10.1016/0278-4343(86)90061-0

LASSO-ALCALÁ, O.M. \& POSADA, J.M. 2010. Presence of the invasive red lionfish, Pterois volitans (Linnaeus, 1758), on the coast of Venezuela, southeastern Caribbean Sea. Aquatic Invasions 5(1):53-59. http://dx.doi. org/10.3391/ai.2010.5.S1.013

LESSA, R \& NÓBREGA, M.F. 2000. Guia de Identificação de Peixes Marinhos da Região Nordeste. Programa REVIZEE / SCORE-NE, p.1-138.

LIMA, A.T., COSTA, P.A.S., BRAGA, A.C., NUNAN, G.W.A. \& MINCARONE, M.M. 2011. Fishes of the family Sternoptychidae (Stomiiformes) collected on the Brazilian continental slope between $11^{\circ}$ and $23^{\circ}$ S. Zootaxa 2741:34-48.

LIMA, A.T. \& MINCARONE, M.M. 2004. Occurrence of Cottunculus granulosus Karrer, 1968 (Scorpaeniformes: Psychrolutidae) in southern Brazil. Comun. Mus. Cienc. Tecnol. PUCRS, Ser. zool. 17(1):39-43.

LUIZ, O.J., FLOETER, S.R., ROCHA, L.A., \& FERREIRA, C.E.L. 2013. Perspectives for the lionfish invasion in the South Atlantic: Are Brazilian reefs protected by the currents? Mar. Ecol. Prog. Ser. 485:1-7.

LUIZ, O.J., MADIN, J.S., ROBERTSON, D.R., ROCHA, L.A., WIRTZ, P. \& S.R. FLOETER. 2012. Ecological traits influencing range expansion across large oceanic dispersal barriers: insights from tropical Atlantic reef fishes. Proc. R. Soc. B. 279: 1033-1040.

LUNDBERG, J.G., MARSHALL, L.G., GUERRERO, J., HORTON, B., MALABARBA, M.C.S.L. \& F. WESSELINGH. 1998. The stage for Neotropical fish diversification: a history of tropical South American rivers. In Phylogeny and classification of Neotropical fishes (L.R. Malabarba, R.E. Reis, R.P. Vari, Z.M.S. Lucena \& C.A.S. Lucena, eds.). EdiPUCRS, p.13-48.

MARCENIUK, A.P. 2005. Redescrição de Genidens barbus (Lacépède, 1803) e Genidens machadoi (Miranda-Ribeiro, 1918), Bagres Marinhos (Siluriformes, Ariidae) do Atlântico Sul Ocidental. Pap. Avulsos de Zool. 45(11):111-125.

MARCENIUK, A.P. 2007. Revalidação de Cathorops arenatus (Valenciennes, 1840) e Cathorops agassizii (Eigenmann \& Eigenmann, 1888) (Siluriformes, Ariidae), bagres marinhos das regiões norte e nordeste da América do Sul. Iheringia, Sér. Zool. 97:360-375. http://dx.doi. org/10.1590/S0073-47212007000400002

MARCENIUK, A.P. \& MENEZES, N.A. 2007. Systematics of the family Ariidae (Ostariophysi, Siluriformes), with a redefinition of thegenera. Zootaxa 1416:1-126.

MARQUES, A.C. \& LAMAS, C.J.E. 2006. Taxonomia zoológica no Brasil: estado da arte, expectativas e sugestões de ações futuras. Pap. avulsos zool. 46(13):139-174.

MARTINS-JURAS, I.A.G.M., JURAS, A.A. \& MENEZES, N.A. 1987. Relação preliminar dos peixes da ilha de São Luís, Maranhão, Brasil. Ver. Bras. Zoo. 4(2):105-113.

MCKENNA, S. \& ALLEN, G.R. 2002. Coral reef biodiversity: assessment and conservation. In Implications for coral reef management and policy: relevant findings from the 19th International Coral Reef Symposium (B.A. Best, R.S. Pomeroy \& C.M. Balboa, eds.). U.S. Agency for International Development, World Resources Institute, Conservation International, International Society for Reef Studies, p.92-94.

MELO, M.R.S. 2007. A new synaphobranchid eel (Anguilliformes: Synaphobranchidae) from Brazil, with comments on the species from the western South Atlantic. Copeia 315-323. http://dx.doi.org/10.1643/00458511(2007)7[315:ANSEAS]2.0.CO;2

MELO, M.R.S., NUNAN, G.W.A., BRAGA, A.C. \& COSTA, P.A.S. 2009. The deep-sea Anguilliformes and Saccopharyngiformes (Teleosei: Elopomorpha) collected on the Brazilian continental slope, between $11^{\circ}$ and $23^{\circ} \mathrm{S}$. Zootaxa 2234:1-20.

MELO, M.R.S., BRAGA, A.C., NUNAN, G.W.A. \& COSTA, P.A.S. 2010. On new collections of deep-sea Gadiformes (Actinopterygii: Teleostei) from the Brazilian continental slope, between $11^{\circ}$ and $23^{\circ} \mathrm{S}$. Zootaxa 2433:25-46

MENEZES, N.A., BUCKUP, P.A., FIGUEIREDO, J.L. \& MOURA, R.L. 2003. Catálogo das espécies de peixes marinhos do Brasil. Universidade de São Paulo, São Paulo, 159p. 
MILLER, P.S. 1995. Selective breeding programs for rare alleles: examples from the Przewalski's horse and California condor pedigrees. Conserv. Biol. 9:1262-1273. http://dx.doi.org/10.1046/j.15231739.1995.9051244.x-i1

MILLIMAN, J.D. \& MEADE, R.H. 1983. World-wide delivery of river sediment to the oceans. J. Geol. 91(1):1-21. http://dx.doi. org $/ 10.1086 / 628741$

MINCARONE, M.M. 2000. Eptatretus menezesi, a new species of hagfish (Agnatha, Myxinidae) from Brazil. B. Mar. Sci. 67(2):815-819.

MINCARONE, M.M. 2001. Myxine sotoi, a new species of hagfish (Agnatha, Myxinidae) from Brazil. B. Mar. Sci. 68(3):479-483.

MINCARONE, M.M. \& SAMPAIO, C.L.S. 2004. First record of the hagfish Eptatretus multidens Fernholm \& Hubbs, 1981 (Myxinidae) in Brazilian waters. Comun. Mus. Cienc. Tecnol. PUCRS, Ser. zool. 17(1):33-38.

MINCARONE, M.M. \& SMITH, D.G. 2005. First record of the grey conger Conger esculentus Poey, 1861 (Congridae) in Brazilian waters. Comun. Mus. Cienc. Tecnol. PUCRS, Ser. zool. 18(1):59-62.

MINCARONE, M.M. \& ANDERSON, M.E. 2008. A new genus and species of eelpout (Teleostei: Zoarcidae) from Brazil. Zootaxa 1852:65-68.

MINCARONE, M.M., NIELSEN, J.G. \& COSTA, P.A.S. 2008. Deep-sea ophidiiform fishes collected on the Brazilian continental slope, between 11 and 23 S. Zootaxa 1770:41-64.

MORA, C., TITTENSOR, D.P. \& MYERS, R.A. 2008. The completeness of taxonomic inventories for describing the global diversity and distribution of marine fishes. P. Roy. Soc. 275:149-155. http://dx.doi.org/10.1098/ rspb.2007.1315

MOURA, R.L. 1995. A new species of Chromis (Perciformes: Pomacentridae) from the southeastern coast of Brazil, with comments on other species of the genus. RFAH 21(3-4):91-96.

MOURA, R.L., FIGUEIREDO, J.L. \& SAZIMA, I. 2001. A new parrotfish (Scaridae) from Brazil, and revalidation of Sparisoma amplum (Ranzani, 1842), Sparisoma frondosum (Agassiz, 1831), Sparisoma axillare (Steindachner, 1878) and Scarus trispinosus Valenciennes, 1840. B. Mar. Sci. 68(3):505-524.

MYERS, N., MITTERMEIER, R.A., MITTERMEIER, C.G., FONSECA, G.A.B. \& KENT, J. 2000. Biodiversity hotspots for conservation priorities. Nature 403:853-858. http://dx.doi.org/10.1038/35002501

NASH, W.G., WIENBERG, J., FERGUSON-SMITH, M.A., MENNINGER, J.C. \& O’BRIEN, S.J. 1998. Comparative genomics: Tracking chromosome evolution in the family Ursidae using reciprocal chromosome painting. Cytogenet. Cell Genet. 83:182-192. http://dx.doi. org/10.1159/000015176

NEIVA, G.S. \& MOURA, S.J.C. 1977. Sumário sobre exploração de recursos marinhos do litoral brasileiro: situação atual e perspectivas. Programa de Pesquisa e Desenvolvimento Pesqueiro do Brasil. Série Documentos Ocasionais, Brasília, 27, p.1-48.

NELSON, G.J. \& PLATNICK, N.I. 1981. Systematics and Biogeography: Cladistics and Vicariance. Columbia University Press, New York.

NIELSEN, J.G. 2009 A revision of the bathyal genus Luciobrotula (Teleostei, Ophidiidae) with two new species. Galathea Report 22:141-156.

O'BRIEN, S.J., ROELKE, M.F., MARKER, L., NEWMAN, A., WINKLER, C.A., MELTZER, D., COLLY, L., EVERMANN, J.F., BUSH, M. \& WILDT, D.E. 1985. Genetic Basis for species vulnerability in the cheetah. Science 227:1428-1434. http://dx.doi.org/10.1126/science.2983425

PINHEIRO, H.T., GASPARINI, J.L. \& SAZIMA, I. 2010. Sparisoma rocha, a new species of parrotfish (Actinopterygii: Labridae) from Trindade Island, south-western Atlantic. Zootaxa 2493:59-65.

RIBEIRO, A.L.R. 2007. Ocorrência e distribuição da ictiofauna estuarina brasileira. Monografia de Especialização em Biologia Marinha, Departamento de Biologia, Universidade de Taubaté, p.1-87.
ROCHA, L.O.F. \& COSTA, P.A.S. 1999. Manual de Identificação de Peixes Marinhos para a Costa Central. Programa REVIZEE / SCORE-Central, p.1-70.

ROCHA, L.A. \& ROSA, I.S. 2001. Baseline assessment of reef fish assemblages of Parcel Manuel Luiz Marine State Park, Maranhão, northeast Brazil. J. Fish Biol. 58:985-998.

SAZIMA, I., MOURA, R.L. \& ROSA, R.S. 1997. Elacatinus figaro sp. n. (Perciformes: Gobiidae), a new cleaner goby from the coast of Brazil. Aqua 2(3):33-38.

SAZIMA, I., GASPARINI, J.L. \& MOURA, R.L. 1998. Gramma brasiliensis, a new basslet from the western South Atlantic (Perciformes: Grammatidae). Aqua 3(1):39-43.

SAZIMA, I., CARVALHO-FILHO, A.\& SAZIMA, C. 2008. A new cleaner species of Elacatinus (Actinopterygii: Gobiidae) from the Southwestern Atlantic. Zootaxa 1932:27-32.

SAZIMA, I., CARVALHO-FILHO, A., GASPARINI, J.L. \& SAZIMA, C. 2009. A new species of scaly blenny of the genus Labrisomus (Acanthopterygii: Labrisomidae) from the tropical West Atlantic. Zootaxa 2015:62-68.

SCHOFIELD, P.J. 2009. Geographic extent and chronology of the invasion of non-native lionfish (Pterois volitans [Linnaeus 1758] and P. miles [Bennett 1828]) in the Western North Atlantic and Caribbean Sea. Aquatic Invasions 4(3): 473-479.

SOTO, J.M.R. 2001a. Galeus mincaronei sp. nov. (Carcharhiniformes, Scyliorhinidae), a new species of sawtail catshark from southern Brazil. Mare Magnum 1(1):11-18

SOTO, J.M.R. 2001b. Schroederichthys saurisqualus sp. nov. (Carcharhiniformes, Scyliorhinidae), a new species of catshark from southern Brazil, with further data on Schroederichthys species. Mare Magnum 1(1):37-50.

SOTO, J.M.R. \& VOOREN, C.M. 2004. Hydrolagus matallanasi sp. nov. (Holocephali, Chimaeridae) a new species of rabbitfish from southern Brazil. Zootaxa 687:1-10.

SPALDING, M.D., FOX, H.E., ALLEN, G.A., DAVIDSON, N., FERDAÑA, Z.A., FINLAYSON, M., HALPERN, B.S., JORGE, M.A., LOMBANA, A., LOURIE, S.A., MARTIN, K.D., MCMANUS, E., MOLNAR, J., RECCHIA, C.A. \& ROBERTSON, J. 2007. Marine ecoregions of the world: a bioregionalization of coastal and shelf areas. BioScience 57:573583. http://dx.doi.org/10.1641/B570707

SULLIVAN SEALEY, K. \& BUSTAMANTE, G. 1999. Setting geographic priorities for marine conservation in Latin America and the Caribbean. The Nature Conservancy, Arlington.

UNESCO. 1988. Challenges for marine science training and education worldwide. UNESCO Rep. Mar. Sci. 52.

VASKE JUNIOR, T., LESSA, R.P., NÓBREGA, M., MONTEALEGREQUIJANO, S., SANTANA, F.M. \& BEZERRA, J.L.Jr. 2004. A checklist of fishes from Saint Peter and Saint Paul Archipelago, Brazil. J.Appl. Ichthyol. 20, p. 1-5.

VAZZOLER, A.E.A.M., SOARES, L.S.H. \& CUNNINGHAM, P.T.M. 1999. Ictiofauna da costa brasileira. In: Estudos ecológicos de comunidades de peixes tropicais. R.H. Lowe-McConnell. Edusp, São Paulo, p.424-467.

WALPOLE, M.J., MORGAN-DAVIES, M., MILLEDGE, M., BETT, P. \& LEADER-WILLIAMS, N. 2001. Population dynamics and future conservation of a free-ranging black rhinoceros (Diceros bicornis) population in Kenya. Biol. Conserv. 99:237-243. http://dx.doi. org/10.1016/S0006-3207(00)00219-6

WOLFF, M., KOCH, V. \& ISAAC, V.J. 2000. A Trophic Flow Model of the Caete Mangrove Estuary (North Brazil) with Considerations for the Sustainable Use of its Resources. Estuar. Coast. Shelf S. 50:789-803. http://dx.doi.org/10.1006/ecss.2000.0611 